\title{
AVALIAÇÃO FÍSICO-QUÍMICA DAS PROPRIEDADES INTERFACIAIS DE FILMES MOLECULARES DE CARVACROL E FOSFOLIPÍDEOS
}

\author{
G.H.R.C. SILVA ${ }^{1}$, F.S. ROCHA ${ }^{1}$, M.D.L. OLIVEIRA ${ }^{1}$ e C.A.S. ANDRADE ${ }^{1}$ \\ ${ }^{1}$ Universidade Federal de Pernambuco, Centro de Ciências Biológicas, Departamento de \\ Bioquímica
}

E-mail para contato: gustavocodeceira@hotmail.com

\begin{abstract}
RESUMO - A nanotecnologia farmacêutica vem se consolidando como alternativa aos tratamentos tradicionais devido às vantagens terapêuticas apresentadas. A técnica de Langmuir é eficiente para a análise de filmes monomoleculares interfaciais fornecendo subsídios para o desenvolvimento de sistemas de liberação controlada efetivos. Dentre as drogas conhecidas o carvacrol é um bioativo com grande utilidade terapêutica devido a suas propriedades antimicrobianas, antioxidantes e anti-inflamatórias, no entanto, seu uso é limitado devido sobretudo à insolubilidade aquosa e baixa biodisponibilidade, sendo uma alternativa sua inclusão em lipossomas, que são capazes de carrear drogas polares e apolares, potencializar a farmacocinética e farmacodinâmica e minimizar os efeitos colaterais. O presente trabalho buscou avaliar a estabilidade das monocamadas mistas compostas por carvacrol e os fosfolipídios DPPE, DPPG e DPPC utilizando-se o método de Langmuir para mensuração das propriedades fisico-químicas. As isotermas sugeriram uma melhor interação às composições envolvendo carvacrol/DPPC devido particularmente às rápidas transições para filmes em estado sólido em baixas pressões e através dos resultados dos parâmetros termodinâmicos foi possível determinar que as composições de carvacrol/DPPC apresentaram maior atratividade intermolecular, logo, sugere-se que tal composição é a mais indicada para a confecção de nanocarreadores dentre os fosfolipídeos estudados.
\end{abstract}

Palavras-chave: Langmuir, Filmes interfaciais, carvacrol

\section{INTRODUÇÃO}

O interesse para o desenvolvimento de novas drogas vem aumentando com o passar dos anos em decorrência da disponibilidade de novas tecnologias. Uma das finalidades é maximizar os efeitos terapêuticos e minimizar efeitos colaterais, como também aumentar biodisponibilidade. No entanto, a produção esbarra no alto custo, no andamento lento e geralmente apresenta prognóstico indeterminado, sendo uma alternativa o uso de drogas preexistentes em sistemas com a mesma finalidade, como em sistemas de liberação controlada, exemplificando, temos os nanocarreadores lipossomais. O carvacrol é um bioativo com grande utilidade terapêutica devido a suas propriedades antimicrobianas, antioxidantes e anti-inflamatórias, no entanto, seu uso é limitado devido sobretudo à 
insolubilidade aquosa e baixa biodisponibilidade, sendo uma alternativa sua inclusão em lipossomas, pois são biodegradáveis, compostos por moléculas anfifílicas, portanto, capazes de transportar compostos polares e apolares. Ademais, oferece proteção ao fármaco, adesão a tecidos e mantém constante a liberação do bioativo, maximizando efeitos terapêuticos e minimizando efeitos colaterais (AKBARZADEH et al, 2013).

A estabilidade do lipossoma depende de suas propriedades físico-químicas e estas podem ser determinadas pelos parâmetros termodinâmicos, tais como energia livre de Gibbs e energia livre de mistura (NASCIMENTO-FILHO et al, 2010). O presente trabalho buscou avaliar a estabilidade das monocamadas mistas compostas por carvacrol e os fosfolipídios DPPE, DPPG e DPPC utilizando-se o método de Langmuir para mensuração das propriedades físico-químicas.

\section{MATERIAIS E MÉTODOS}

O carvacrol (1,2-benzopirona) e os fosfolipídios Dipalmitoilfostatidilglicerol (DPPG), Dipalmitoilfosfatidiletanolamina (DPPE) e Dipalmitoilfosfatidilcolina (DPPC) foram adquiridos da Sigma-Aldrich (St. Louis, MO, USA). Foram preparadas soluções estoque de cumarina/lipídios em diferentes frações molares $(\times=0.25,0.375,0.5,0.675,0.75$ e 0.875$)$ diluídas em clorofórmio na concentração de $1 \mathrm{mg} \cdot \mathrm{mL}^{-1}$. Posteriormente, foram dispersos 10 $\mu \mathrm{L}$ dessas frações com o auxílio de uma micropipeta (Hamilton 701n), em uma subfase contendo $20 \mathrm{~mL}$ de água deionizada (Millipore-Synergy) apresentando resistividade de 18.2 $\mathrm{M} \Omega . \mathrm{cm}$ e pH 7.0. Depois de 15 minutos de espera, para garantir a evaporação do solvente, a compressão foi iniciada com o deslocamento simétrico das barreiras em velocidade constante de $20 \mathrm{~mm} / \mathrm{min}$ na temperatura de $23 \pm 0.2^{\circ} \mathrm{C}$. As medidas de pressão de superfície $(\pi)$ foram realizadas utilizando uma cuba de Langmuir (KIBRON, Finlândia) equipada com um sistema de medida pelo método da placa de Wilhelmy, com mensuração da pressão de superfície apresentando acurácia de $\pm 0,1 \mathrm{mN} / \mathrm{m}$. Os resultados foram coletados usando o software FilmWare X da KIBRON (LUNA et al., 2011; ANDRADE, 2006).

\section{RESULTADOS E DISCUSSÃO}

Figura 1. Pressão de superfície dos filmes mistos de carvacrol e fosfolipídios.

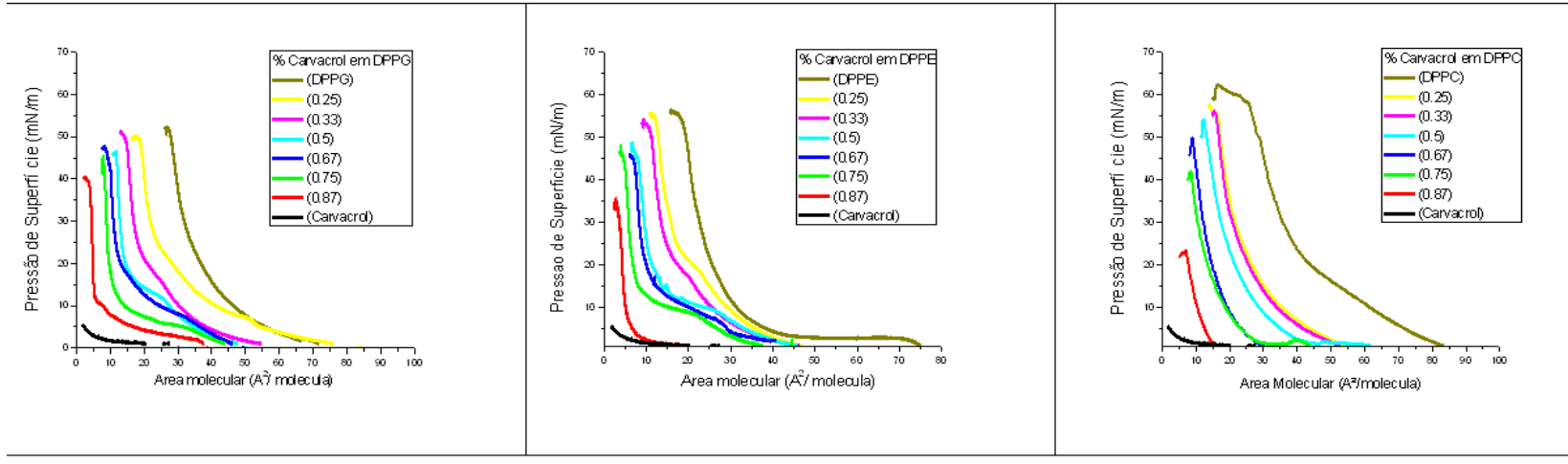

O sistema envolvendo carvacrol/DPPG apresentou um perfil expandido em baixas pressões, observando-se uma transição para uma fase sólida apenas em pressões maiores que $10 \mathrm{mN} . \mathrm{m}^{-1}$, para as frações estudadas. Após a interação do carvacrol com o DPPE é observado um perfil similar ao ocorrido com o fosfolipídio anterior, em baixas pressões, com característica de filme expandido onde, em seguida, houve uma transição para um filme sólido, exceto a fração de 0.87 , que apresentou rápida transição em baixa pressão. Por outro 
lado, o filme misto de carvacrol/DPPC apresentou de início, rápida transição para um filme sólido a partir da fração 0.25 , sendo mais evidentemente notado a partir da fração 0.67 (Fig. 1).

Figura 2. Energia livre de Gibbs dos filmes mistos de carvacrol e fosfolipídios.

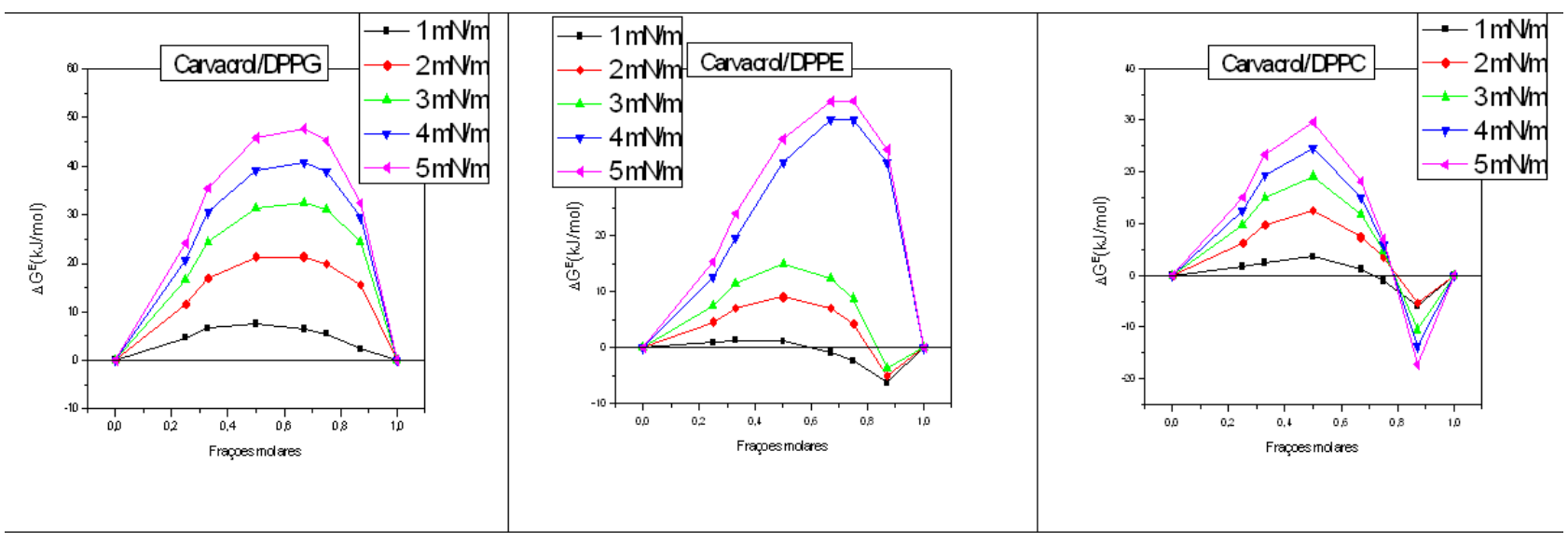

Figura 3. Energia livre de mistura dos filmes mistos de carvacrol e fosfolipídios.
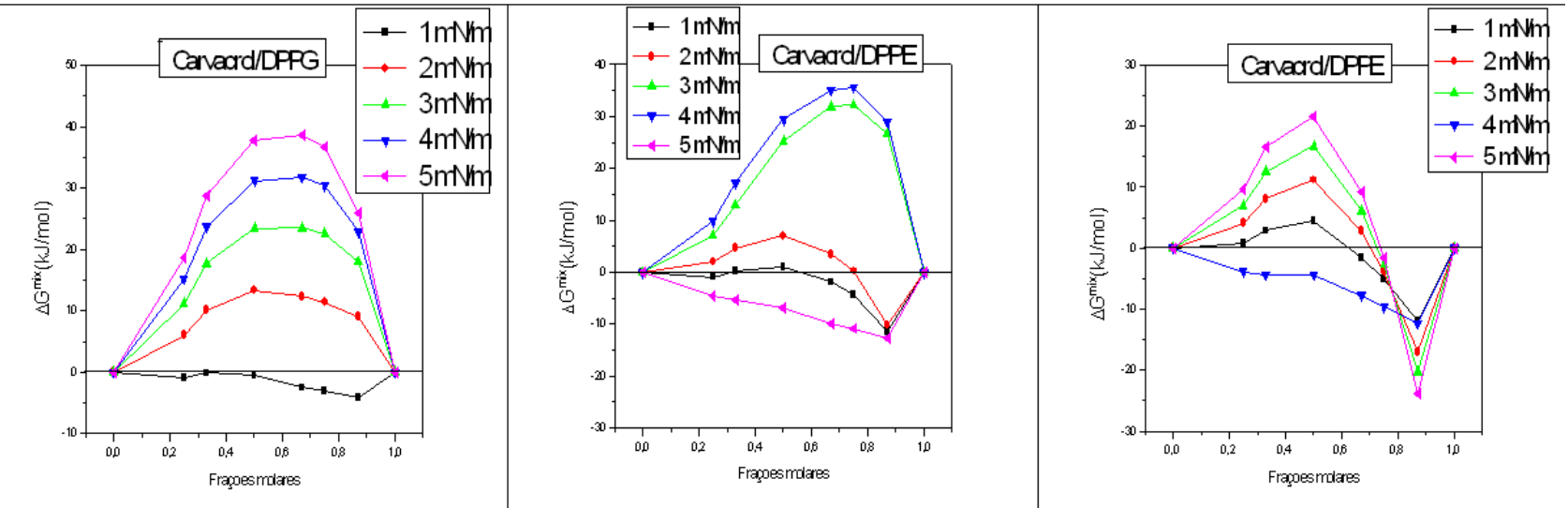

Verificou-se nos estudos termodinâmicos de energia livre de Gibbs e de mistura interação favorável em diferentes graus entre carvacrol e os demais lipídios nas proporções estudadas.

Porém, nas monocamadas mistas de carvacrol/DPPG, notou-se que, independente da pressão de superfície estudada, as interações intermoleculares foram menos significativas devido às interferências das regiões polares entre o agrupamento hidroxila do carvacrol $\left(\mathrm{OH}^{-}\right)$e a região polar do DPPG $\left(\mathrm{OH}^{-}\right)_{2}$. Soma-se a isso, a formação de agregados de carvacrol em proporções maiores de droga devido às interações desfavoráveis com o DPPG no sistema, indicadas pela resultante positiva da entalpia. Logo, as moléculas das composições de carvacrol/DPPG apresentaram caráter repulsivo, especialmente em pressões maiores que $1 \mathrm{mN} . \mathrm{m}^{-1}$, causando aumento da entropia e repulsão no sistema. No entanto, nos filmes compostos por carvacrol/DPPC e carvacrol/DPPE observou-se um comportamento atrativo, sobretudo com maior proporção de droga em pressões abaixo de $5 \mathrm{mN} . \mathrm{m}^{-1}$, 
supostamente em consequência da região polar protonada $\left(\mathrm{N}^{+}\right.$e $\mathrm{NH}_{3}{ }^{+}$de DPPC e DPPE respectivamente) apresentada pelos fosfolipídios DPPE e DPPC, tendo este último uma grande área molecular, favorecendo uma maior interação com o carvacrol e uma diminuta formação de agregados. Portanto, os estudos sinalizaram um sistema estável devido à baixa entropia e quando há uma elevada proporção de droga no sistema. Dentre as misturas favoráveis estudadas, o filme composto por carvacrol/DPPC na fração de $\mathrm{XCa}=$ 0.87 mostrou-se como a melhor composição, particularmente quando submetida a uma baixa pressão $\left(\pi=5 \mathrm{mN} . \mathrm{m}^{-1}\right)$, resultado de uma baixa entropia e melhor interação molecular (Figs. 2 e 3 ).

\section{CONCLUSÃO}

Conforme os resultados de variação de energia livre de Gibbs e energia livre de mistura, os filmes compostos por cumarina/DPPG e carvacrol/DPPC na fração de $(\mathrm{X}=$ 0.87) mostraram uma maior estabilidade em relação as demais composições estudadas. Logo, sugere-se que estas composições são as mais indicadas para a construção de nanocarreadores, visando uma maior eficiência no tratamento terapêutico.

\section{REFERÊNCIAS}

AKBARZADEH, A; REZAEI-SADABADY, R; DAVARAN, S. Liposome: classification, preparation, and applications. Nanoscale Research Letters. 2013;8(1):102

NASCIMENTO-FILHO, J. M.; DE MELO, C. P.; SANTOS-MAGALHÃES, V. R.; MACIEL, M. A. M.; ANDRADE, C. A. S.; Colloids Surf., A. 2010, 358, 42.

LUNA, D.M.N; FALCAO E.P.S.; MELO S.J.; ANDRADE C.A.S. Interfacial properties of a novel pyrimidine derivative and poly(ethylene glycol)-grafted phospholipid floating monolayers. Coll Surf A. 2011;373:22-8

ANDRADE, C.A.S.; SANTOS-MAGALHAES, N.S.; MELO, C.P.D. Thermodynamic characterization of the prevailing molecular interactions in mixed floating monolayers of phospholipids and usnic acid. J. Colloid Interface Sci., 298 (2006), pp. 145-153

\section{AGRADECIMENTOS}

À Facepe - Fundação de amparo a ciência e tecnologia do estado de Pernambuco. 